\title{
Microstructure Evolution and Phase Transformation of Ti-6.5Al-2Zr-Mo-V Alloy During Thermohydrogen Treatment
}

\author{
Hong-Chao Kou $\cdot$ Han-Lei Zhang $\cdot$ Yu-Dong Chu $\cdot$ Dong Huang $\cdot$ Hai Nan $\cdot$ Jin-Shan Li
}

Received: 11 August 2014/Revised: 15 September 2014/Published online: 10 February 2015

(C) The Chinese Society for Metals and Springer-Verlag Berlin Heidelberg 2015

\begin{abstract}
Thermohydrogen treatment (THT) is an effective way to refine microstructure and improve the mechanical properties of the titanium alloys. In the current work, as-cast Ti-6.5Al-2Zr-Mo-V alloy was hydrogenated with different hydrogen contents and processed solution aging. Accordingly, the microstructure evolution and phase transformation were analyzed. Results show that during solution aging, eutectoid decomposition occurs and the product is a mixture of coarse primary $\alpha$, fine eutectoid product and undecomposed $\beta_{\mathrm{H}}$. The size of primary $\alpha$ is closely dependent on the hydrogen content, and large primary $\alpha$ can be obtained at medium hydrogen content. Further, the influence of hydrogen content on the growth of primary $\alpha$ phase was revealed. The primary $\alpha$ is much fine, and the eutectoid product is relatively homogeneous with $0.984 \mathrm{wt} \% \mathrm{H}$. After THT, the ultimate strength is beyond 1,100 MPa that has increased by $23.15 \%$ compared with that in as-cast state.
\end{abstract}

KEY WORDS: Titanium alloy; Hydrogen; Heat treatment; Phase transformation; Eutectoid decomposition; Mechanical property

\section{Introduction}

As a chemical heat treatment [1], thermohydrogen treatment (THT) is an effective way to refine the microstructure [2] and improve the mechanical properties of the titanium alloys [3]. By this method, the microstructure can be refined without deformation and recrystallization [4], so it is especially suitable for castings that do not allow deformation. During THT, the hydrogen is added into the alloy and removed at high temperatures in vacuum [5]. Since the reaction of

Available online at http://link.springer.com/journal/40195

H.-C. Kou · H.-L. Zhang ( $ه)$ · Y.-D. Chu · J.-S. Li

State Key Laboratory of Solidification Processing, Northwestern

Polytechnical University, Xi' an 710072, China

e-mail: zhlnwpu4181@mail.nwpu.edu.cn

D. Huang $\cdot$ H. Nan

Beijing Institute of Aeronautical Materials, Beijing 100095,

China hydrogen with titanium is reversible [6], hydrogen acts as a temporary alloying element and does not modify the chemical composition of the titanium alloys [7].

A typical THT process is composed of three specific steps: hydrogenation, solution-aging treatment (eutectoid decomposition) and dehydrogenation [8]. The solution aging acts as a major step of refinement, during which eutectoid decomposition $\beta_{\mathrm{H}} \rightarrow \alpha+\delta$ occurs and fine, acicular product precipitates, which is beneficial to the mechanical properties [9]. However, undecomposed $\delta$ hydride and coarse heterogeneous primary $\alpha$ phase also form during the solution aging, which is detrimental to the mechanical properties [10]. They may generate embrittlement of the alloy, which is a major restriction for the application of THT [11]. To obtain a better combination of the mechanical properties, the $\delta$ hydride should be eliminated and the size of the primary $\alpha$ should be restricted. Studies show [12] that the size of primary $\alpha$ is closely dependent on the hydrogen content, but the detailed mechanisms remain unknown. The current work focuses on the effect of the hydrogen content on 
the size of primary $\alpha$, that is growth of primary $\alpha$ in the aged microstructure.

The Ti-6.5Al-2Zr-Mo-V alloy is widely applied in aeronautic turbines due to its low density and excellent hightemperature performance (up to $500{ }^{\circ} \mathrm{C}$ ) [13]. In the current work, Ti-6.5Al-2Zr-Mo-V was hydrogenated with different hydrogen contents and then processed with solution aging. Microstructure and XRD patterns were obtained at the end of hydrogenation and solution aging. By analyzing the aged microstructure, the relationship between the hydrogen content and the size of primary $\alpha$ solution aging was established. Based on this relationship, the influence of the hydrogen content on the growth of primary $\alpha$ was revealed.

\section{Materials and Methods}

Ti-6.5Al-2Zr-Mo-V alloy used in the current study was obtained from Beijing Institute of Aeronautical materials (China). The as-received alloy is the rods prepared by investment casting, and its chemical composition is shown in Table 1. The rods were machined into cylindrical samples for THT. Photographs of the as-cast rod and the cylindrical sample are shown in Fig. 1a. During THT, the hydrogenation was performed in a vacuum annealing furnace, in an atmosphere of hydrogen, at $750{ }^{\circ} \mathrm{C}$ for $2 \mathrm{~h}$. Figure $1 \mathrm{~b}$ shows the diagram of hydrogenation and heat treatment processes. After hydrogenation, the solution treatment was carried out in the $\beta$ field for $0.5 \mathrm{~h}$, followed by aging at $600{ }^{\circ} \mathrm{C}$ for $8 \mathrm{~h}$; then, the samples were quenched in water (WQ) to retain the microstructure as shown in Fig. 1b. By controlling the pressure of hydrogen, samples with different $\mathrm{H}$ contents of $0.259,0.430,0.569,0.820$ and $0.984 \mathrm{wt} \%$ were obtained. These hydrogen contents are marked in a schematic binary Ti-H diagram from Ref. [14], as shown in Fig. 1c.

The hydrogen content was determined with an electric balance with accuracy of $0.01 \mathrm{mg}$. The microstructure was observed by an OLYMPUS GX51 optical microscopy (OM) and a VEGA II LMH scanning electric microscope (SEM). The phase composition was analyzed by a DX2700 X-ray diffractometer (XRD). An FEI Tecnai G2 F30 transmission electron microscope (TEM) was used to analyze the microstructure and acquire the selected-area diffraction pattern (SADP). The tensile test was performed using a MTS SANS CMT5105 machine.

\section{Results}

\subsection{Hydrogenation and the Microstructure}

Figure 2 shows XRD patterns of the as-cast microstructure and the hydrogenated microstructures. Since Ti-6.5Al-
$2 \mathrm{Zr}-\mathrm{Mo}-\mathrm{V}$ is a near $\alpha$ alloy [15], the as-cast microstructure is composed of a large amount of $\alpha$ phase and little $\beta$ phase. In the hydrogenated microstructures, $\delta$ hydride precipitates and $\beta$ transforms into $\beta_{\mathrm{H}}$ ( $\beta$ containing hydrogen).

When the hydrogen content increases, formation of $\delta$ and $\beta_{\mathrm{H}}$ is promoted [16], and their concentration increases. The increase in the hydrogen content also influences the lattice parameters of $\beta_{\mathrm{H}}$ phase. Because $\mathrm{H}$ atoms occupy interstitial positions in the lattice of $\beta_{\mathrm{H}}$ [17], a higher hydrogen concentration in $\beta_{\mathrm{H}}$ increases its lattice parameters, and the diffraction peaks of $\beta / \beta_{\mathrm{H}}$ phase gradually move to a lower angle.

Figure 3 presents optical micrographs of the as-cast microstructure and the hydrogenated microstructure $(0.984 \mathrm{wt} \% \mathrm{H})$. As shown in Fig. 3a, the as-cast microstructure is a typical lamellar structure [18-20]. The white portion represents $\alpha$ phase, and the gray is $\beta$ phase. The size of the former $\beta$ grain is about $500 \mu \mathrm{m}$ in average, and lamellar colonies precipitate in the $\beta$ grains.

Compared with the as-cast microstructure, the hydrogenated microstructure (Fig. 3b) also contains lamellar colonies formed inside the $\beta$ grains, but the morphology is much different. The lamellar microstructure is fragmented in some local regions, and some coarse black regions can be observed, which is considered to be a mixture of $\beta_{\mathrm{H}}$ phase and $\delta$ hydride. The precipitation of $\beta_{\mathrm{H}}$ and $\delta$ is preferred on the grain boundaries and the lamella interfaces, where the atomic lattice is distorted [11]. The distortion and interfacial energy on the $\beta$ grain boundary is much higher than those of the lamella interface [17], so there is more precipitation on the $\beta$ boundaries. This precipitation is harmful to the ductility of the alloy. Not only the $\delta$ hydride itself is very brittle [8], but also the high concentration of hydrogen may reduce the ductility of $\alpha$ phase [9]. Compared with the alloys without hydrogen, this precipitation may act as source of brittleness. Morphology of the $\beta$ boundary is also changed in the hydrogenated microstructure, which transforms from straight into a zigzag shape, a phenomenon that has not been reported.

\subsection{Microstructure at the End of Aging}

Figure 4 shows XRD patterns at the end of aging. Compared with Fig. 2, the concentration of remaining $\beta_{\mathrm{H}}$ phase is much lower. This is because $\beta_{\mathrm{H}}$ decomposes much more completely during aging $[21,22]$. In some cases, the decomposition is not complete [23, 24], so the microstructure is less homogeneous and the mechanical properties are undermined.

The concentration of remaining $\beta_{\mathrm{H}}$ phase varies with different hydrogen contents (Fig. 4). Hydrogen content influences the $\beta_{\mathrm{H}}$ decomposition in a complex way. The increase in the hydrogen facilitates precipitation of $\delta$ 
Table 1 Chemical compositions of the as-cast Ti-6.5Al-2Zr-Mo-V alloy (wt\%)

\begin{tabular}{llllllllllll}
\hline $\mathrm{Ti}$ & $\mathrm{Al}$ & $\mathrm{Zr}$ & $\mathrm{Mo}$ & $\mathrm{V}$ & $\mathrm{Fe}$ & $\mathrm{Si}$ & $\mathrm{C}$ & $\mathrm{N}$ & $\mathrm{H}$ & $\mathrm{O}$ & $\mathrm{Other}$ \\
\hline Balance & 6.0 & 2.0 & 1.7 & 2.2 & 0.04 & 0.05 & 0.02 & 0.01 & 0.001 & 0.14 & 0.30 \\
\hline
\end{tabular}

a
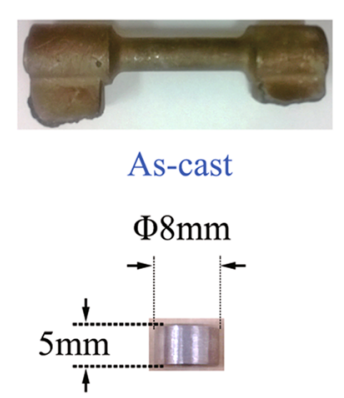

After machining b Hydrogenation

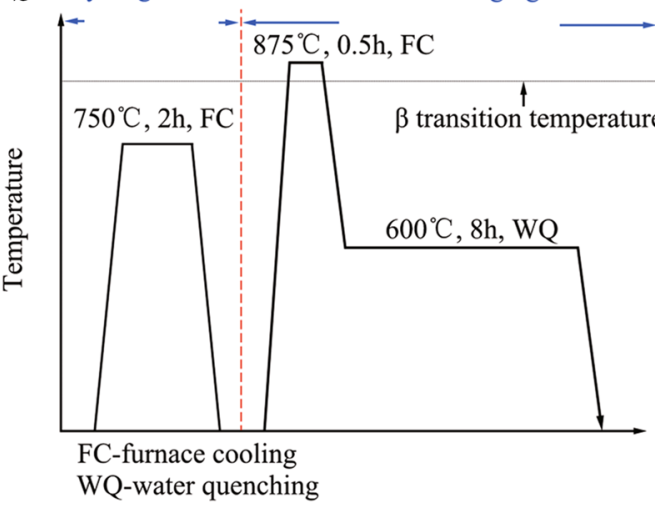

c

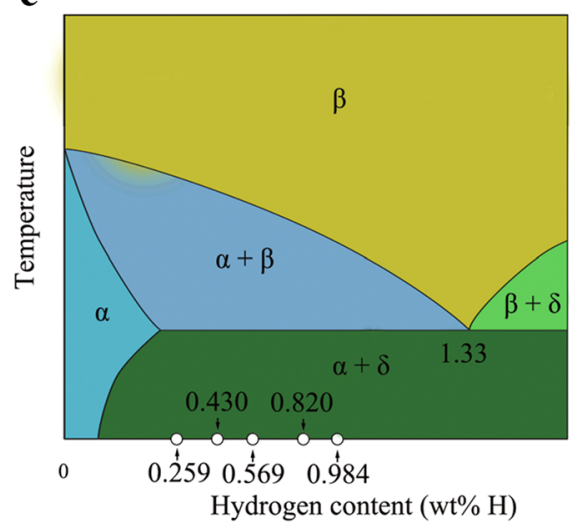

Fig. 1 a Photographs of the as-cast rod and the cylindrical sample, $\mathbf{b}$ hydrogenation and solution aging, $\mathbf{c}$ various hydrogen contents obtained during hydrogenation

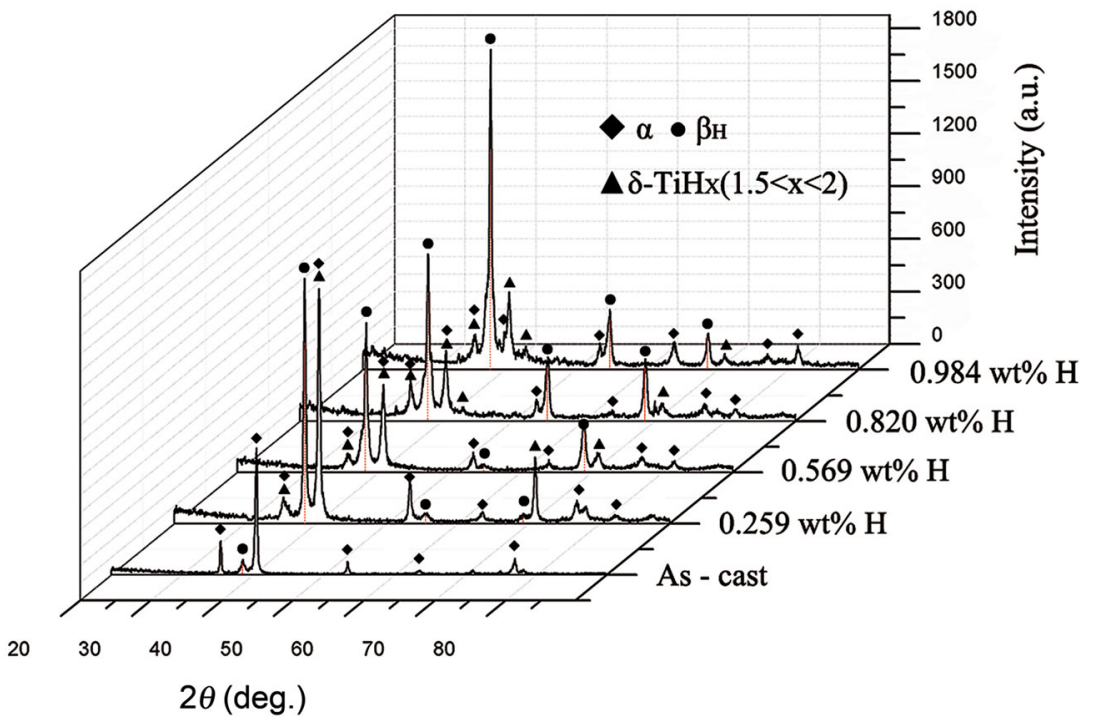

Fig. 2 XRD patterns of the as-cast microstructure and the hydrogenated microstructures

hydride, which is a product of the eutectoid decomposition, so the eutectoid decomposition would also be more complete, and the concentration of $\beta_{\mathrm{H}}$ becomes lower. In a different perspective, the addition of the hydrogen may reduce the $\beta$ transition temperature, which means the decomposition of $\beta_{\mathrm{H}}$ is inhibited [25, 26], and the concentration of $\beta_{\mathrm{H}}$ should be high as a result. It seems that the increase in the hydrogen content can both reduce and raise the content of $\beta_{\mathrm{H}}$. This complicated mechanism will be discussed in detail in the Discussion section.

Figure 5 shows SEM graphs of the aged microstructures. During the process of aging, eutectoid decomposition $\beta_{\mathrm{H}} \rightarrow \alpha+\delta$ takes place, and the product is closely dependent on the hydrogen content. Since the hydrogen content in the current work is not at the eutectoid point of Ti-H system, primary $\alpha$ or $\delta$ should precipitate first, and 
then, eutectoid product ( $\alpha$ and $\delta$ ) precipitates between the primary phases $[27,28]$. The primary phase is coarse needles, while the eutectoid product is fine needles. As can be seen, there is no significant segregation of $\delta$ hydride, which is detrimental to the mechanical properties. Since the $\delta$ hydride distributes homogeneously in the matrix, it is easy to remove during the dehydrogenation. The effect of the hydrogen content on aged microstructure is presented in Fig. 5a-e.

Figure 5a shows aged microstructure with $0.259 \mathrm{wt} \% \mathrm{H}$. Because this hydrogen content is too low, the microstructure is not primary phase and eutectoid product, but close to the lamellar structure. $\alpha$ Lamella is $1-2 \mu \mathrm{m}$ in width and $20-30 \mu \mathrm{m}$ in length. There is also some secondary $\alpha$ phase, which forms due to a low nucleation rate of $\alpha$ phase. Since hydrogen is a $\beta$ stabilizer, the addition of hydrogen may reduce the $\beta$ transition temperature. With reducing $\beta$ transition temperature, undercooling is also reduced and the nucleation rate of $\alpha$ is inhibited [29, 30], which leads to the formation of secondary $\alpha$ [31].

With $0.430 \mathrm{wt} \% \mathrm{H}$ (Fig. 5b), the microstructure is majorly composed of a large amount of eutectoid product and little primary phase. The eutectoid product is homogeneous and slender, which is about $0.1 \mu \mathrm{m}$ in width and 10-20 $\mu \mathrm{m}$ in length. Some tiny primary phases precipitate on the $\beta$ grain boundaries, which are the preferential locations for nucleation. Width of the primary phase is about $1 \mu \mathrm{m}$, which is coarser than that of the eutectoid product. In Fig. $5 c$, d, large primary phase distributes on the matrix of fine eutectoid product. The eutectoid product is similar to that in Fig. $5 \mathrm{~b}$, but the primary phase is much coarser, which is $2-4 \mu \mathrm{m}$ in width and $10-40 \mu \mathrm{m}$ in length. In Fig. 5e, there are small primary phases and fine eutectoid products, which is similar to Fig. $5 b$, but its primary phase is even finer.

It is seen from Fig. 5b-e, larger primary phase can be obtained at medium hydrogen content, and smaller ones
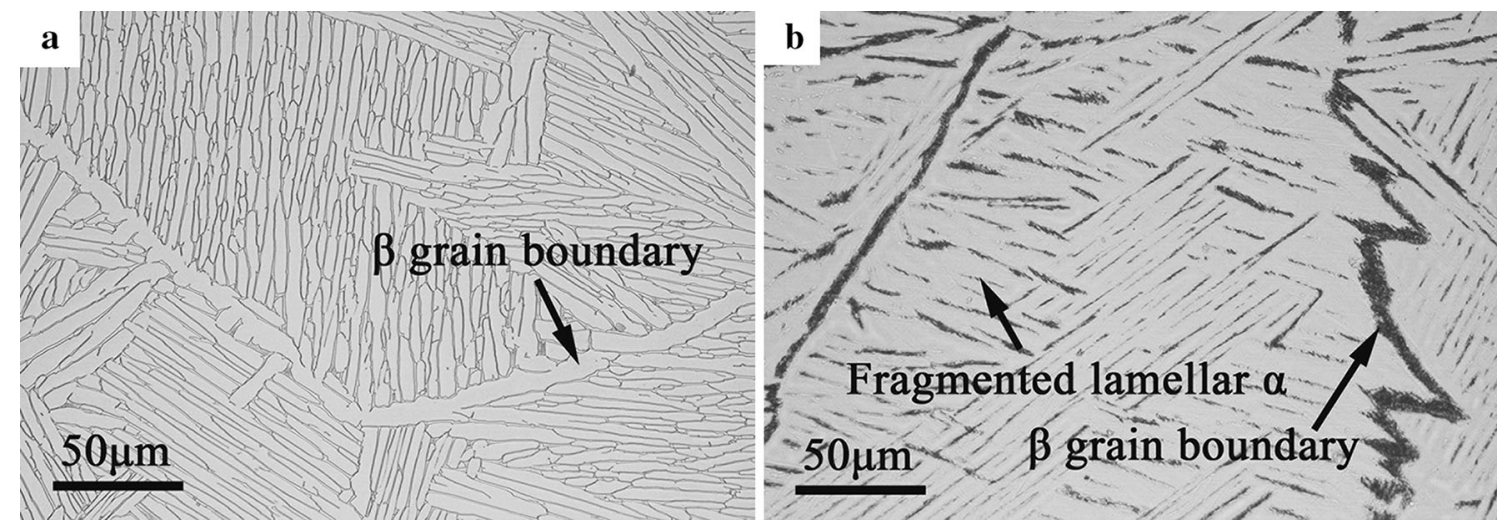

Fig. 3 Optical micrographs of the as-cast microstructure a and the hydrogenated microstructure $(0.984 \mathrm{wt} \% \mathrm{H}) \mathbf{b}$

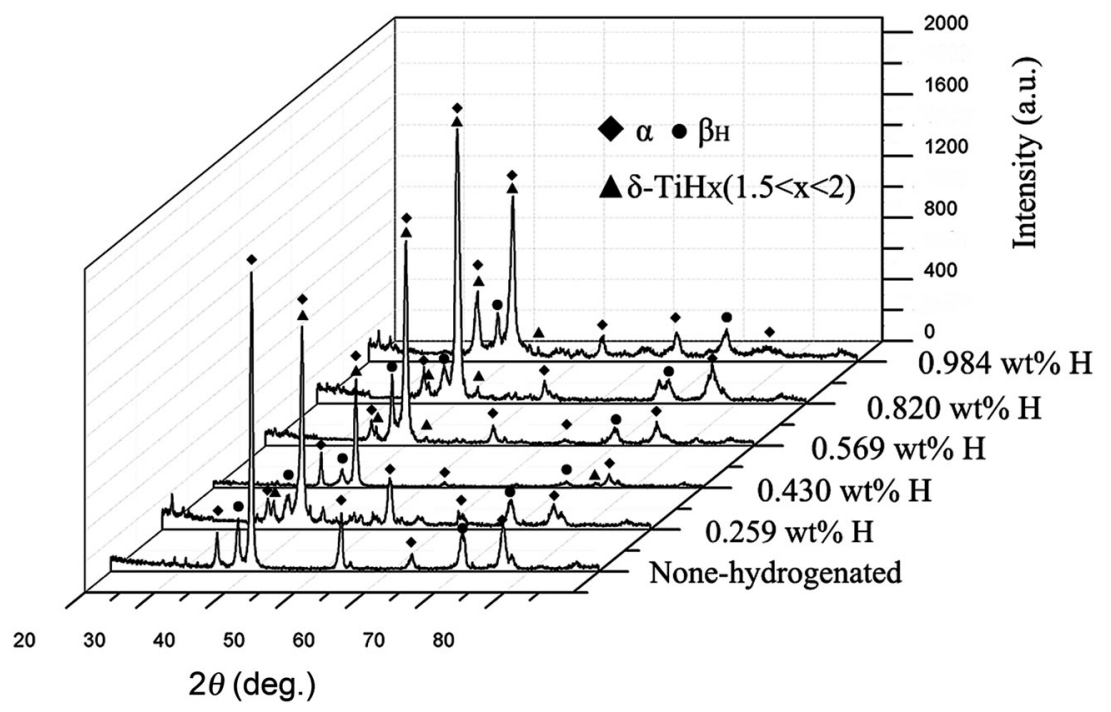

Fig. 4 XRD patterns of the aged microstructures 
occur at both low and high hydrogen contents. According to the Ti-H binary diagram, the size of the primary phase would decrease with the increasing hydrogen [22], so the current phenomenon cannot be simply explained by $\mathrm{Ti}-\mathrm{H}$ binary diagram. A detailed study of this phenomenon will be presented in Sect. 4.

Analysis of Fig. 5 shows that the aged microstructure consists of the primary phase, and eutectoid product precipitates between these primary phases. The eutectoid product is a mixture of acicular $\alpha$ and $\delta$, and the primary phase can be either $\alpha$ or $\delta[11,25]$. In Ti-H system, the eutectoid point is $1.33 \mathrm{wt} \% \mathrm{H}$ [14], and in our work, the highest hydrogen content is $0.984 \mathrm{wt} \% \mathrm{H}$, which suggests that the primary phase should be $\alpha$ phase. However, some studies show that the primary $\delta$ hydride can precipitates with a quite low hydrogen content $(0.885 \mathrm{wt} \% \mathrm{H})[8,25]$. To identify the primary phase in the current work, the primary phase with $0.984 \mathrm{wt} \% \mathrm{H}$ is dealt with some further TEM observation.

Figure 6a is a TEM graph of the aged microstructure $(0.984 \mathrm{wt} \% \mathrm{H})$, which contains a lot of primary phase. To identify the primary phase, a coarse primary needle and a fine primary needle are selected and SADPs are obtained, as shown in Fig. 6b, c. By indexing the SADPs, it can be found out that Fig. $6 \mathrm{~b}$ shows a diffraction pattern of $\alpha$ phase from the zone axis of $[1 \overline{1} 0 \overline{3}]$. Figure $6 \mathrm{c}$ also presents a pattern of $\alpha$ phase, but it is from the zone axis of [2200]. Accordingly, the primary phase in Fig. $6 \mathrm{a}$ is $\alpha$ phase, and no primary $\delta$ forms. Since $0.984 \mathrm{wt} \% \mathrm{H}$ is the highest hydrogen content in the current work, the primary phase at lower hydrogen content is also $\alpha$ phase.

According to some studies $[8,25]$, primary $\delta$ hydride precipitates in Ti-6Al-4V with $0.885 \mathrm{wt} \% \mathrm{H}$. In the current work, however, at $0.984 \mathrm{wt} \% \mathrm{H}$, which is much higher, the primary phase is still $\alpha$. This interesting phenomenon may be caused by the higher amount of $\beta$ phase in Ti-6Al- $-4 \mathrm{~V}$. Since $\beta$ phase is a good solvent for hydrogen, hydrogen tends to concentrate in $\beta$, resulting in the fact that the hydrogen concentration in the $\beta_{\mathrm{H}}$ phase is much higher than the average value [32]. When the average hydrogen content is still lower than the eutectoid point, the hydrogen content in $\beta_{\mathrm{H}}$ may exceed the eutectoid point, and $\delta$ hydride could precipitate. In the current $\mathrm{Ti}-6.5 \mathrm{Al}-2 \mathrm{Zr}-\mathrm{Mo}-\mathrm{V}$ alloy, however, there is little $\beta$ phase. With little $\beta$ phase, there is not significant segregation of hydrogen, and the hydrogen is evenly distributed in the alloy. Considering this, $\delta$ hydride cannot form until the average hydrogen content is higher than the eutectoid point.

From one perspective, the formation of primary $\alpha$ may be beneficial to the mechanical properties. Since primary $\alpha$ phase forms instead of primary $\delta$, a source of brittleness is eliminated. However, this effect is highly dependent on the size of the primary $\alpha$. If the primary $\alpha$ grows too large, it is also detrimental to the ductility. The size of the primary $\alpha$ will be discussed in the following section.
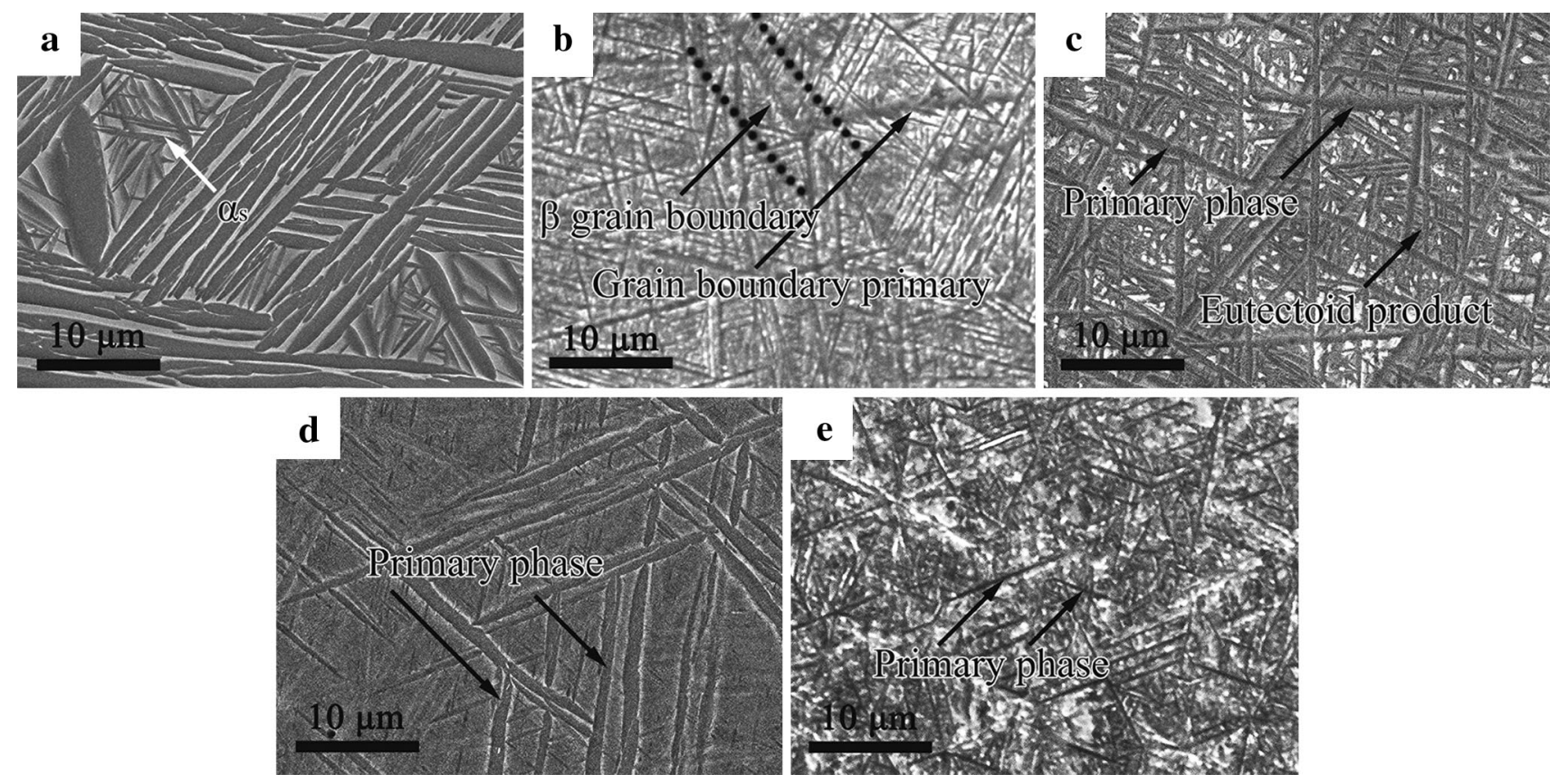

Fig. 5 SEM micrographs of the aged microstructures. a 0.259 wt $\% \mathrm{H}$, b 0.430 wt $\% \mathrm{H}$, c $0.569 \mathrm{wt} \% \mathrm{H}$, d 0.820 wt $\% \mathrm{H}$, e $0.984 \mathrm{wt} \% \mathrm{H}$ 


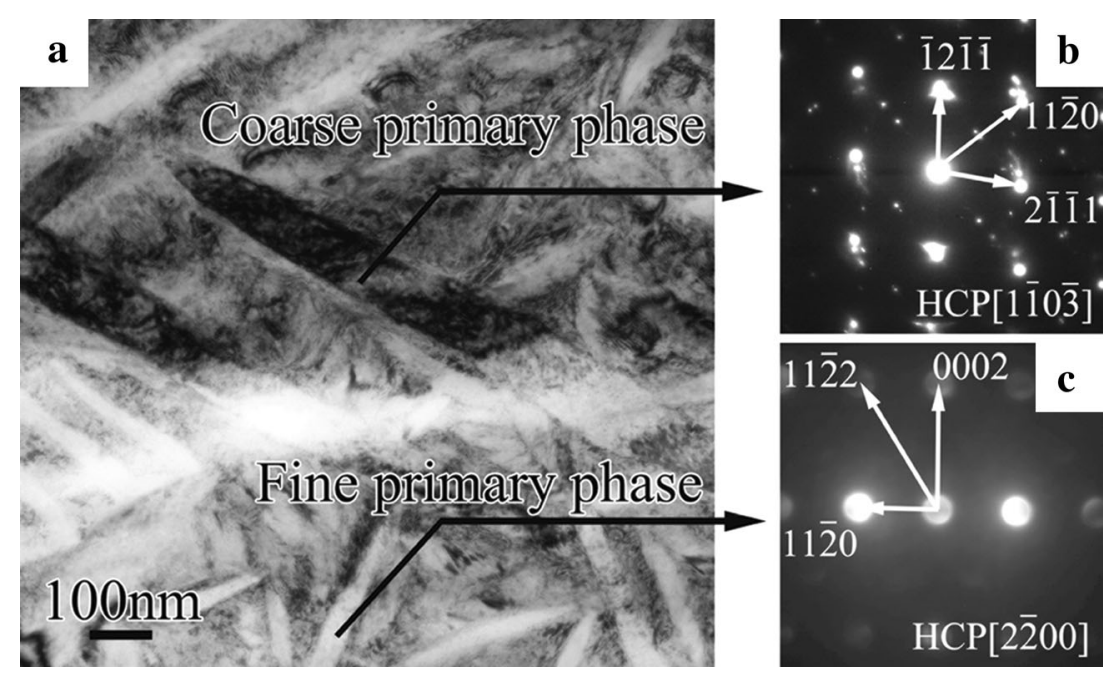

Fig. 6 a TEM micrograph of the aged microstructure (with 0.984 wt $\%$ H), b SADP of a coarse primary phase, $\mathbf{c}$ SADP of a fine primary phase
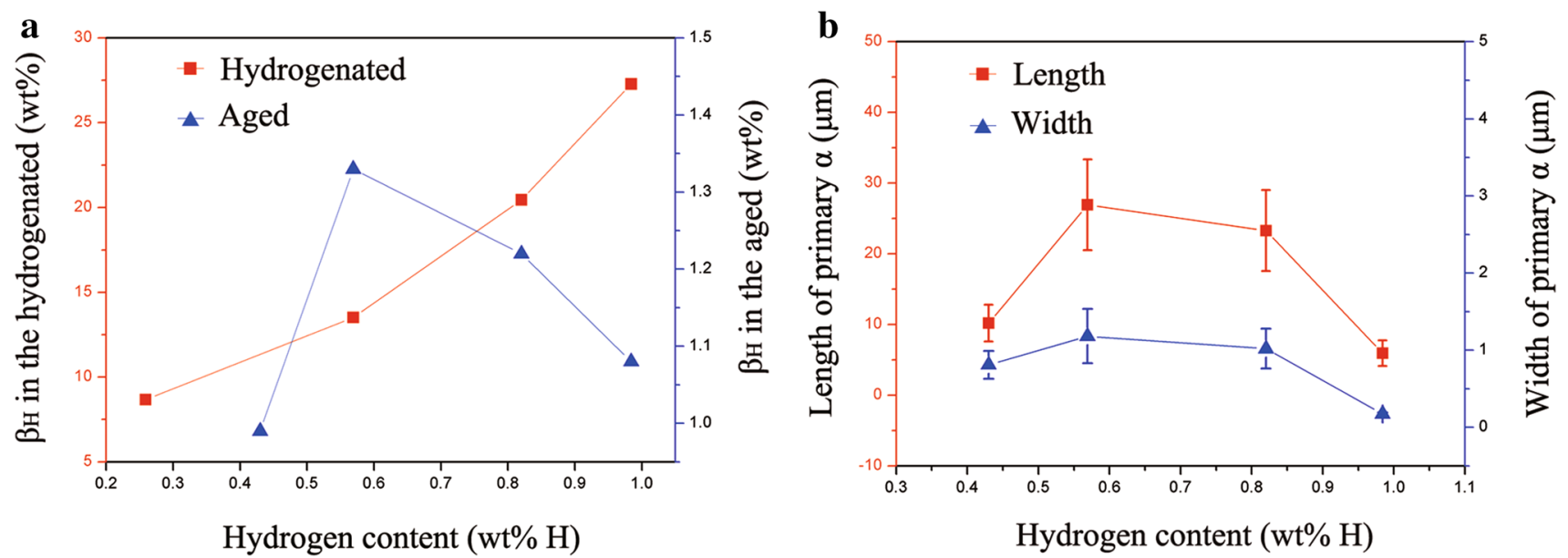

Fig. 7 a Content of $\beta_{\mathrm{H}}$ at the end of hydrogenation and aging, $\mathbf{b}$ size of primary $\alpha$ phase at the end of aging

\section{Discussion}

\subsection{Statistical Analysis of the Microstructure after Aging}

As shown in Figs. 5 and 6, the aged microstructure consists of primary $\alpha$ and a mixture of eutectoid $\alpha$ and $\delta$. There is also some undecomposed $\beta_{\mathrm{H}}$, which is mixed with the eutectoid $\alpha$ and $\delta$. Analysis of the microstructure shows that content of undecomposed $\beta_{\mathrm{H}}$ and the size of primary $\alpha$ change with the hydrogen content, as shown in Fig. 7.

For every single phase in Figs. 2 and 4, the integrated intensity of its peaks has been calculated. Based on the results, the content of every phase can be decided. Figure 7a shows content of $\beta_{\mathrm{H}}$ at the end of dehydrogenation and aging. For the hydrogenated microstructure, $\beta_{\mathrm{H}}$ content increases with hydrogen content. Since hydrogen is a $\beta$ stabilizer, more hydrogen can preserve more $\beta_{\mathrm{H}}$. For the aged microstructure, however, the change in $\beta_{\mathrm{H}}$ content presents a different trend. With low $(0.430 \mathrm{wt} \% \mathrm{H})$ and high $(0.984 \mathrm{wt} \% \mathrm{H})$ hydrogen contents, the content of $\beta_{\mathrm{H}}$ is quite low, which indicates that the decomposition of $\beta_{\mathrm{H}}$ is relatively complete. Differently, at medium hydrogen contents $(0.569$ and $0.820 \mathrm{wt} \%)$, there is a much higher concentration of $\beta_{\mathrm{H}}$, which means that the $\beta_{\mathrm{H}}$ are not completely decomposed.

The length and width of the primary $\alpha$ in the aged microstructure are measured and presented in Fig. 7b. For every individual hydrogen content, length and width of 50 primary $\alpha$ needles were measured to obtain the average length and width. It is interesting that the length and width of primary $\alpha$ have a similar trend with the $\beta_{\mathrm{H}}$ content in the aged microstructure (Fig. 7a). Small primary $\alpha$ forms under low and high hydrogen contents, and large primary $\alpha$ precipitates under medium hydrogen contents. 


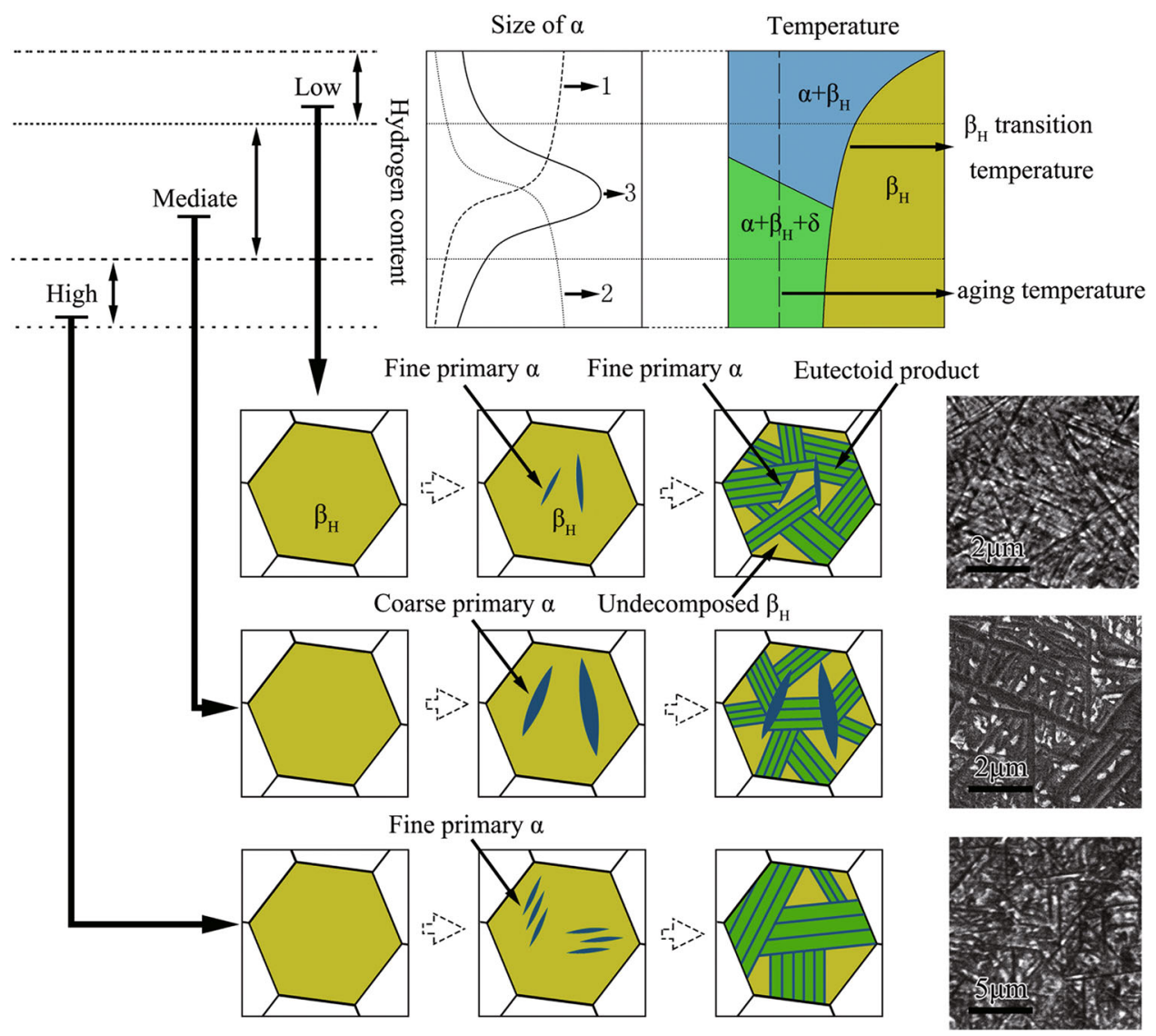

Fig. 8 Effect of hydrogen content on the size of primary $\alpha$ phase. Curve 1 shows effect of undercooling on $\alpha$ size, curve 2 shows effect of eutectoid decomposition, and curve 3 shows sum of the two effects. A pseudo-Ti-H diagram is presented to show undercooling and formation of $\delta$. Formation of primary $\alpha$ at three different hydrogen contents (low, medium and high) is presented. $R_{\mathrm{p} 0.2}$ is yield strength, $R_{\mathrm{m}}$ is ultimate strength, $A$ is unit extension, and $Z$ is reduction of area

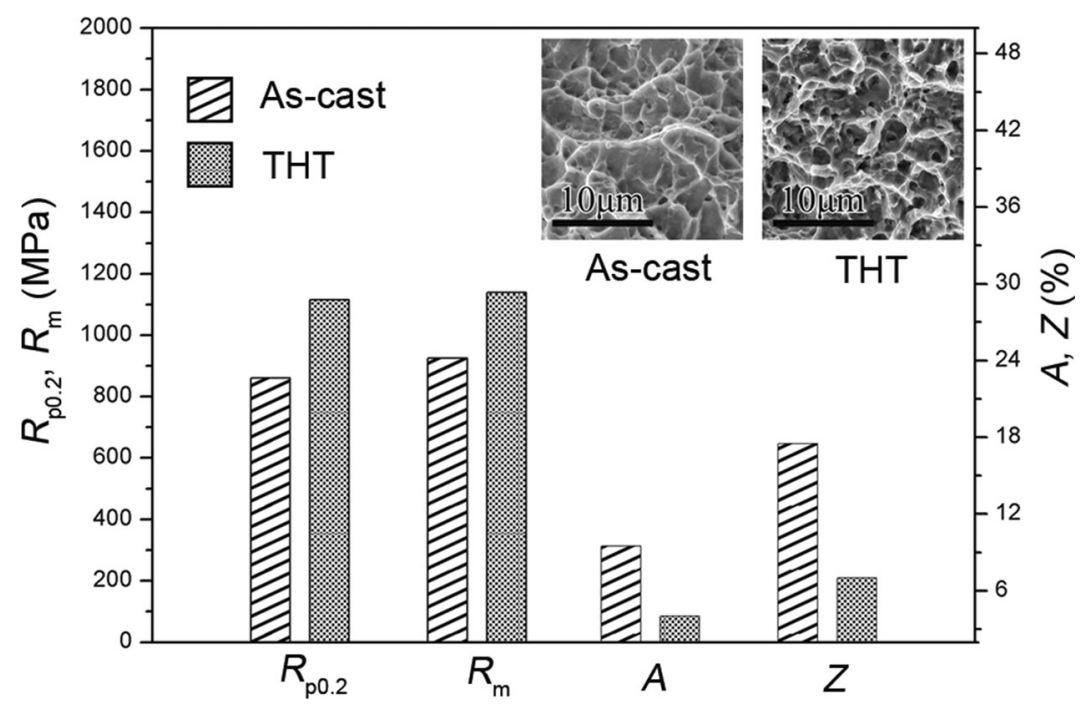

Fig. 9 Tensile properties of the as-cast sample and the sample processed with THT 


\subsection{Growth of Primary $\alpha$}

Analysis of Fig. 7 shows that the size of primary $\alpha$ and the content of $\beta_{\mathrm{H}}$ changes with hydrogen content in the aged microstructure. Larger primary $\alpha$ and more $\beta_{\mathrm{H}}$ can be obtained at medium hydrogen contents $(0.569$ and $0.820 \mathrm{wt} \% \mathrm{H})$. The size of primary $\alpha$ is dependent on transformation $\beta_{\mathrm{H}} \rightarrow \alpha$, and the concentration of $\beta_{\mathrm{H}}$ is decided by the eutectoid decomposition $\beta_{\mathrm{H}} \rightarrow \alpha+\delta$. It is obvious that the relationship between these two transformations is the key to decide the eutectoid microstructure, which is complex. Although some works [33, 34] suggest that the two transformations take place in sequence, another study [22] indicates that they are competitive. The precipitation of primary $\alpha$ could inhibit the eutectoid decomposition, and the eutectoid decomposition also declines the formation of primary $\alpha$.

The effect of the hydrogen content on the size of primary $\alpha$ can be explained with the Ti-H phase graph [14, 35]. When the hydrogen content increases, two mechanisms with opposite effect would influence on the size of primary $\alpha$ : (1) $\beta_{\mathrm{H}}$ transition temperature decreases, so the undercooling and nucleation rate of primary $\alpha$ also declines. Less primary $\alpha$ needles would form, and every individual needle tends to grow larger, and (2) $\delta$ hydride is more likely to precipitate, and the eutectoid decomposition is thus promoted. The eutectoid decomposition is competitive with the formation of primary $\alpha$, so the formation of $\alpha$ is depressed, and the primary $\alpha$ tends to be smaller.

The growth of primary $\alpha$ is determined by the combination of the two mechanisms, so larger primary $\alpha$ should form at medium hydrogen contents. At high and low hydrogen contents, the primary $\alpha$ is small and the microstructure mainly consists of eutectoid product. The combined growth of primary $\alpha$ can be schematically illustrated in Fig. 8. Curve 1 shows the way that hydride precipitation affects the size of primary $\alpha$, and curve 2 shows the effect of undercooling. Curve 3 shows the combined effect of curve 1 and 2. A schematic pseudo-Ti-H graph [28] is also presented to show $\delta$ precipitation and undercooling at different hydrogen contents. Growth of primary $\alpha$ at three different hydrogen contents (low, medium and high) is presented and explained schematically.

With the growth mechanism of primary $\alpha$, the relationship between the $\beta_{\mathrm{H}}$ content and the hydrogen content (Fig. 7a) can be explained as well: At both low and high hydrogen contents, the nucleation of primary $\alpha$ is inhibited and the eutectoid decomposition $\beta_{\mathrm{H}} \rightarrow \alpha+\delta$ is thus promoted. With a more complete eutectoid decomposition, there would be little $\beta_{\mathrm{H}}$ remaining. At the medium hydrogen contents of 0.569 and $0.820 \mathrm{wt} \%$, large primary $\alpha$ phase emerges and eutectoid decomposition is thus depressed. $\beta_{\mathrm{H}}$ is not completely decomposed, and its content remains high.

\subsection{Tensile Properties}

Figure 9 shows that the tensile properties of the as-cast alloy and the alloy dealt with THT $(0.984 \mathrm{wt} \% \mathrm{H}$, aged at $600{ }^{\circ} \mathrm{C}$ for $8 \mathrm{~h}$ and then dehydrogenated at $700{ }^{\circ} \mathrm{C}$ for $6 \mathrm{~h}$ ). Homogeneous dimples can be observed in the fracture topography, which indicates that both as-cast and THT samples part by ductile fracture [36-38]. Compared with that of the as-cast alloy, $R_{\mathrm{p} 0.2}$ and $R_{\mathrm{m}}$ increase 29.65 and $23.15 \%$ after THT, respectively. As studied in Fig. 5, during THT, the lamellar structure of as-cast alloy completely transforms to fine, homogeneous microstructure, which is beneficial to tensile strength. Also, the massive grain boundary $\alpha$ in as-cast state (Fig. 2a) is eliminated after solution aging (Fig. 5). The grain boundary $\alpha$ phase can cause microbrittleness [39-42] and is harmful to the tensile properties, so its elimination is beneficial to the improvement in mechanical properties.

Although strength increases after THT, the value of $A$ and $Z$ decreases, which means a loss of the ductility. This is considered to be caused by the primary $\alpha$ phase formed during eutectoid decomposition [7]. The loss of the ductility may reduce the combined mechanical properties of the alloy, which can further compromise the application of the alloy.

The ductility can be effectively improved by eliminating the primary $\alpha$ or restricting its size during aging. Studies $[22,43]$ show that at a lower aging temperature, the growth of primary $\alpha$ is depressed and its size is smaller. This method does not decline the strength, so a better combination of the mechanical properties can be obtained. However, aging at a lower temperature means the eutectoid decomposition is relatively slow [22], and it would take more time to complete. This may cause problems like oxidation [44] or loss of hydrogen [45]. Therefore, further work should be done to solve these problems.

\section{Conclusions}

In the current work, $\mathrm{Ti}-6.5 \mathrm{Al}-2 \mathrm{Zr}-\mathrm{Mo}-\mathrm{V}$ was processed with two steps of THT: hydrogenation and solution aging. During aging, eutectoid decomposition $\beta_{\mathrm{H}} \rightarrow \alpha+\delta$ hydride takes place, and the microstructure consists of primary $\alpha$ and eutectoid product. The primary $\alpha$ is large and needle-like, and fine acicular eutectoid product precipitates between the primary $\alpha$.

The size of the primary $\alpha$ changes remarkably with different hydrogen contents. Larger primary $\alpha$ can be obtained at medium hydrogen contents, and small primary $\alpha$ precipitates at low and high hydrogen contents. This is because the growth of primary $\alpha$ is inhibited by its high nucleation rate at low hydrogen content, and it is restricted 
by precipitation of $\delta$ hydride at high hydrogen content. With a high hydrogen content of $0.984 \mathrm{wt} \%$, primary $\alpha$ is fine and eutectoid product is homogeneous. After THT, the ultimate strength is beyond 1,100 MPa, which increases by $23.15 \%$ compared with that of the as-cast state.

\section{References}

[1] T.Y. Fang, W.H. Wang, Mater. Chem. Phys. 56, 35 (1998)

[2] F. Froes, O. Senkov, J. Qazi, Int. Mater. Rev. 49, 3 (2004)

[3] O.N. Senkov, F.H. Froes, Thermohydrogen Processing of Titanium Alloys (Elsevier, Kidlington, 1999)

[4] O.N. Senkov, J.J. Jonas, F.H. Froes, JOM 48, 42 (1996)

[5] E. Tal-Gutelmacher, R. Gemma, C.A. Volkert, R. Kirchheim, Scr. Mater. 63, 1032 (2010)

[6] J.I. Qazi, J. Rahim, O.N. Senkov, F.H. Froes, JOM 54, 68 (2002)

[7] Z. Sun, W. Zhou, H. Hou, Int. J. Hydrog. Energy 34, 1971 (2009)

[8] V. Madina, I. Azkarate, Int. J. Hydrog. Energy 34, 5976 (2009)

[9] C.P. Liang, H.R. Gong, Int. J. Hydrog. Energy 35, 3812 (2010)

[10] M. Li, Y. Lin, Int. J. Hydrog. Energy 32, 626 (2007)

[11] Y. Niu, M. Li, Metall. Mater. Trans. A 40, 3009 (2009)

[12] A.A. Il'in, M.V. Gol'tsova, Metall. Sci. Heat Treat. 50, 261 (2008)

[13] C. Wu, H. Yang, H.W. Li, Acta Metall. Sin. (Engl. Lett.) 26, 533 (2013)

[14] F.D. Manchester, Phase Diagrams of Binary Hydrogen Alloys (ASM International, Russell Township, Geauga County, 2000)

[15] Y. Liu, J.C. Zhu, Mech. Mater. 40, 792 (2008)

[16] P.M. Proa-Flores, G. Mendoza-Suarez, R.A.L. Drew, J. Mater. Sci. 47, 455 (2012)

[17] G. Lütjering, J.C. Williams, Titanium (Springer, Berlin, 2007)

[18] Z. Chen, J. Li, J. Liu, Q. Wang, J. Liu, R. Yang, J. Mater. Sci. Technol. 26, 564 (2010)

[19] K. Sanosh, A. Balakrishnan, L. Francis, T. Kim, J. Mater. Sci. Technol. 26, 904 (2010)

[20] Q. Wang, D.L. Sun, X.L. Han, W.G. Wang, Acta Metall. Sin. (Engl. Lett.) 23, 106 (2010)
[21] A.A. Il'in, S.V. Skvortsova, A.M. Mamonov, G.V. Permyakova, D.A. Kurnikov, Metall. Sci. Heat Treat. 44, 185 (2002)

[22] W. Kerr, MTA 16, 1077 (1985)

[23] D.H. Kohn, P. Ducheyne, J. Mater. Sci. 26, 328 (1991)

[24] J. Zhao, H. Ding, Y. Zhong, C.S. Lee, Int. J. Hydrog. Energy 35, 6448 (2010)

[25] T. Zhu, M. Li, Mater. Sci. Eng. A 527, 7080 (2010)

[26] D. Eliezer, N. Eliaz, O.N. Senkov, F.H. Froes, Mater. Sci. Eng. A 280, 220 (2000)

[27] D. Shan, Y. Zong, T. Lu, Y. Lv, J. Alloys Compd. 427, 229 (2007)

[28] H. Liu, L. Zhou, P. Liu, Q. Liu, Int. J. Hydrog. Energy 34, 9596 (2009)

[29] K. Wang, W. Zeng, Y. Zhao, Y. Lai, Y. Zhou, J. Mater. Sci. 45, 5883 (2010)

[30] S. Li, L. Yang, L. Lei, C. Wei, H. Zhang, J. Mater. Sci. Technol. 28, 1015 (2012)

[31] S. Zhu, H. Yang, L.G. Guo, X.G. Fan, Mater. Charact. 70, 101 (2012)

[32] D. Setoyama, J. Matsunaga, H. Muta, M. Uno, S. Yamanaka, J. Alloys Compd. 385, 156 (2004)

[33] H.-J. Christ, M. Decker, S. Zeitler, Metall. Mater. Trans. A 31, $1507(2000)$

[34] L. Luo, Y. Su, J. Guo, H. Fu, J. Alloys Compd. 425, 140 (2006)

[35] L. Zhou, H. Liu, Mater. Charact. 62, 1036 (2011)

[36] X. Ye, J. Kuang, X. Li, G. Tang, J. Alloys Compd. 599, 1 (2014)

[37] S.Q. Wang, J.H. Liu, D.L. Chen, Mater. Des. 56, 174 (2014)

[38] B.Q. Li, X. Lu, Acta Metall. Sin. (Engl. Lett.) 27, 338 (2014)

[39] C.M. Liu, H.M. Wang, X.J. Tian, D. Liu, Mater. Sci. Eng. A 604, 176 (2014)

[40] M. Salib, J. Teixeira, L. Germain, E. Lamielle, N. Gey, E. AebyGautier, Acta Mater. 61, 3758 (2013)

[41] D.H. Lee, W. Nam, J. Mater. Sci. 34, 2843 (1999)

[42] S. Roy, S. Suwas, Mater. Des. 58, 52 (2014)

[43] A. Guitar, G. Vigna, M.I. Luppo, J. Mech. Behav. Biomed. Mater. 2, 156 (2009)

[44] J.H. Luan, Z.B. Jiao, G. Chen, C.T. Liu, J. Alloys Compd. 602, 235 (2014)

[45] T. Zhu, M. Li, Mater. Charact. 62, 724 (2011) 